\title{
HOW THE DEARBORN INTELLIGENCE EXAMINATION STANDARDS WERE OBTAINED
}

\author{
WALTER F. DEARBORN \\ $\triangle N D$ \\ EDWARD A. LINCOLN \\ Psycho-educational Clinic, Harvard University
}

The customary way of standardizing any test is to give it to as many children as possible, and combine the results to get norms which are somewhat impressive because of the large numbers upon which they are based. The theory of this procedure seems to be sound, but in practice it has given rise to some serious difficulties. Complaints have been heard that the results of some of the tests place whole classes very much too high or too low, and that the rankings obtained on two or more tests are sometimes widely different.

In the hope of obviating some of these difficulties a new method was tried in the standardizing of the Dearborn tests. The Series II examinations were given in three towns in every grade from the second through the senior class in the high school. It is hard to say just what a typical American town is, but the towns selected do not seem specialized in any way. In each of them agriculture is carried on to considerable extent, but each also does considerable manufacturing. They are large enough to support fairly large numbers of small business men, and are near enough to Boston so that the large city is a fairly open field for the inhabitants. There is in each town a fair sprinkling of children of foreign parents.

The scores were not lumped, but the results from each community were treated separately. They were distributed by months, so that it was possible to find not only the median score for the pupils of each year, but the median age as well. It has heretofore been the assumption that the children of a certain age have a median exactly at the half year, that is, for example, the children from 13.0 to 13.99 years old have a median of 13.5 years. This supposition was found to be incorrect in relation to the pupils studied for these standards. In one community the median 11 year old was only 11.33 , and there were many smaller variations.

When the median scores and ages were obtained they were plotted as in the accompanying diagram. On this diagram points were chosen at each half year for standards. These were taken with the 
attempt to make such standards that the median child of each age should have an intelligence quotient within the normal group $(0.90$ to 1.10) no matter which community he was in. This criterion is very admirably fulfilled. In the twelfth year, where the discrepancies are the greatest, the median child in the lowest scoring group has an IQ of 0.93 , and the median child in the highest scoring group has 1.04 for an IQ. The other deviations from 1.00 are much smaller.

It is very likely that classes, schools, and possibly one or two school systems will be found in which the distribution of intelligence quotients will be rather decidedly skewed in one way or the other.

It is believed, however, that in most of these cases the reason for the skew will be apparent. The authors have found for example, that in the foreign section of a city where the adults are engaged mostly in unskilled or semi-skilled labor the intelligence quotients on both group and individual examinations are likely to run low. It frequently appears, as may be seen on the accompanying diagram, that the pupils of a certain age or a certain grade are out of line with what seems to be the general tendency of the pupils in the community.

Series I was standardized in the same way as Series II, although it was not practicable to get results from so many upper grade children, and thus the standards from the twelfth year on had to be estimated somewhat from the continuation of the lines at their upper ends.

This method is especially valuable in that it exposes facts which are concealed when results are thrown together, and thus more intelligent treatment of the data is possible. 
90

80

10 .

60 苞

50

40

30

20

.

8

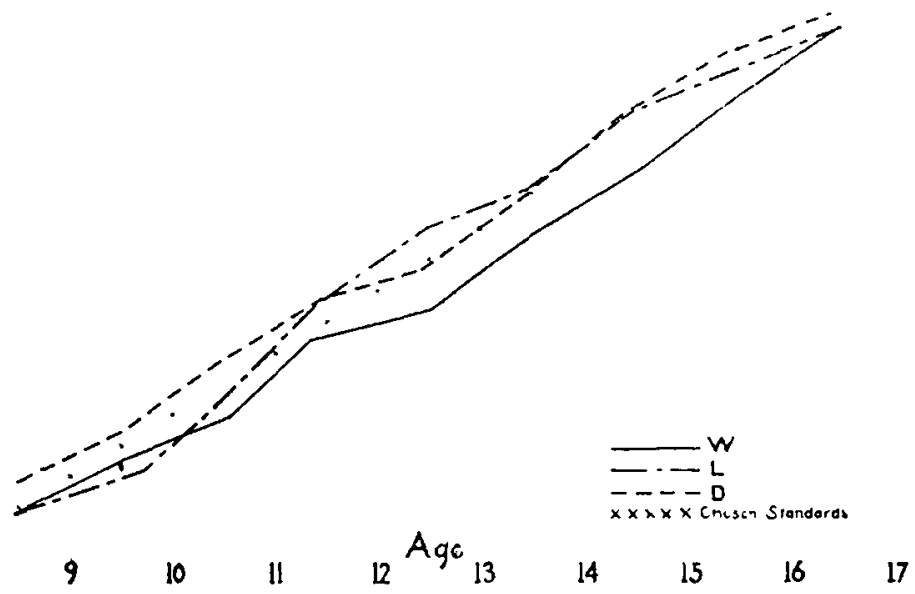

